\title{
A counterexample concerning the variance-optimal martingale measure
}

\author{
Aleš Černý \\ Jan Kallsen \\ City University London* TU München ${ }^{\dagger}$
}

\begin{abstract}
The present note addresses an open question concerning a sufficient characterization of the variance-optimal martingale measure. Denote by $S$ the discounted price process of an asset and suppose that $Q^{\star}$ is an equivalent martingale measure whose density is a multiple of $1-\varphi \cdot S_{T}$ for some $S$-integrable process $\varphi$. We show that $Q^{\star}$ does not necessarily coincide with the variance-optimal martingale measure, not even if $\varphi \cdot S$ is a uniformly integrable $Q^{\star}$-martingale.
\end{abstract}

Key words: Variance-optimal martingale measure, dual characterization

Mathematics Subject Classification (2000): 91B28, 60H05, 93E20

JEL Classification Code: G11, G12, C61

\section{Introduction}

Suppose that the continuous semimartingale $S$ denotes the discounted price process of some asset and fix a time horizon $T \in(0, \infty]$. An equivalent martingale measure $Q^{\star}$ is called variance-optimal martingale measure (VOMM) if its density $\frac{d Q^{\star}}{d P}$ has minimal variance among all equivalent martingale measures. Here, equivalent martingale measure (EMM) refers to probability measures $Q \sim P$ such that $S$ is a $Q$-local martingale. The VOMM plays a key role for mean-variance hedging in incomplete markets (cf. Pham 2000; Schweizer 2001). Its existence is guaranteed under the following mild assumption (cf. Delbaen and Schachermayer 1996).

Assumption 1.1 Process $S$ admits an EMM $Q$ with $E\left(\left(\frac{d Q}{d P}\right)^{2}\right)<\infty$.

*Cass Business School, City University London, 106 Bunhill Row, London EC1Y 8TZ, United Kingdom, (e-mail: cerny@ martingales.info)

${ }^{\dagger}$ HVB-Stiftungsinstitut für Finanzmathematik, Zentrum Mathematik, TU München, Boltzmannstraße 3, 85747 Garching bei München, Germany, (e-mail: kallsen@ma.tum.de) 
In this paper we are concerned with criteria for practical determination of the VOMM. The following dual characterization of the VOMM has been observed and applied repeatedly (see Schweizer 1994; Delbaen and Schachermayer 1996; Delbaen et al. 1997; Mania and Tevzadze 2003).

Proposition 1.2 Let $Q^{\star} \sim P$ denote some EMM with square-integrable density, i.e. with $\frac{d Q^{\star}}{d P} \in L^{2}(P)$. Then $Q^{\star}$ is the VOMM if and only if there exists some S-integrable process $\varphi$ (with finite limit $\varphi \cdot S_{\infty}$ if $T=\infty$ ) such that

1.

$$
\frac{d Q^{\star}}{d P}=\frac{1-\varphi \cdot S_{T}}{E\left(1-\varphi \cdot S_{T}\right)}
$$

2. $\varphi$ is admissible in the sense that $\varphi \cdot S$ is a uniformly integrable $Q$-martingale for any EMM $Q$ with square-integrable density.

PROOF. $\Rightarrow$ : This is shown in this form in Delbaen and Schachermayer (1996), Lemma 2.2.

$\Leftarrow$ : We recall the simple proof for future reference. Note that $E_{Q}\left(\frac{d Q^{\star}}{d P}\right)=\frac{1}{E\left(1-\varphi^{\bullet} S_{T}\right)}$ for any EMM $Q$ with square-integrable density. This implies

$$
\begin{aligned}
E\left(\left(\frac{d Q}{d P}\right)^{2}\right) & =E\left(\left(\frac{d Q^{\star}}{d P}\right)^{2}\right)+2 E\left(\frac{d Q^{\star}}{d P}\left(\frac{d Q}{d P}-\frac{d Q^{\star}}{d P}\right)\right)+E\left(\left(\frac{d Q}{d P}-\frac{d Q^{\star}}{d P}\right)^{2}\right) \\
& =E\left(\left(\frac{d Q^{\star}}{d P}\right)^{2}\right)+2 E_{Q}\left(\frac{d Q^{\star}}{d P}\right)-2 E_{Q^{\star}}\left(\frac{d Q^{\star}}{d P}\right)+E\left(\left(\frac{d Q}{d P}-\frac{d Q^{\star}}{d P}\right)^{2}\right) \\
& \geq E\left(\left(\frac{d Q^{\star}}{d P}\right)^{2}\right)
\end{aligned}
$$

for any such $Q$.

This result can be extended to discontinuous processes if one allows for signed rather than positive measures (cf. Černý and Kallsen 2005) but we consider only continuous price processes in the present paper. The integrand $\varphi$ in Proposition 1.2 minimizes $E((1-\varphi \cdot$ $\left.S_{T}\right)^{2}$ ) among all admissible strategies. In that sense, it corresponds to an optimal quadratic hedging strategy for the constant claim 1 given initial endowment 0 . Alternatively, it can be interpreted as an efficient strategy in a Markowitz sense, i.e. there exists no other strategy with at most the same variance yielding a higher expected return.

The literature has generated a number of alternative characterizations of the VOMM that on the surface look very different from each other and also from Proposition 1.2. But they all share the basic structure of Proposition 1.2: They contain i) a recipe for constructing a candidate measure, and ii) a verification procedure to check that the candidate measure is the true VOMM. We review and unify the different approaches in Section 3. For the time being we will illustrate the main idea in the setup of Proposition 1.2.

Admissibility of a given integrand may be hard to verify. One easily shows that Condition 2 of Proposition 1.2 can in fact be replaced by the seemingly weaker condition

2'. $\varphi \cdot S$ is a uniformly integrable $Q$-martingale for the VOMM $Q$. 
Proof. $1-\varphi \cdot S$ is nonnegative because it is a $Q$-martingale with positive final value. Since it is a local $Q^{\star}$-martingale, it is a $Q^{\star}$-supermartingale as well, which in turn implies $E_{Q^{\star}}\left(\frac{d Q^{\star}}{d P}\right) \leq \frac{1}{E\left(1-\varphi^{\bullet} S_{T}\right)}$. The same reasoning as in (1.2) now yields that the density of $Q^{\star}$ does not have larger variance than the density of the VOMM $Q$.

In applications, however, this modification does not help much. Typically one determines a candidate measure $Q^{\star}$ whose density is of the form (1.1). But since one has no prior knowledge of the true VOMM, proving 2' is not easier than proving 2.

Considering the difficulty of verifying Condition 2 and the circular nature of Condition 2 ' one is naturally lead to investigate the validity of the following

\section{Claim 1.3 Proposition 1.2 holds with Condition 2 replaced by}

\section{$2 " . \varphi \cdot S$ is a uniformly integrable $Q^{\star}$-martingale.}

Put differently, uniform integrability of the local martingale $\varphi \cdot S$ is merely supposed to hold relative to the candidate measure. Since this candidate measure is often known explicitly, such a result would be extremely useful for verification. Furthermore, Condition 2" implies that the candidate martingale measure $Q^{\star}$ has a square-integrable density as required by Assumption 1.1, hence no further computations would be needed.

The main contribution of this paper is in showing that Claim 1.3 fails to hold, that is one cannot replace the unpleasant condition 2 with the more amenable condition 2". Since all $P$-martingales are continuous in our counterexample, it shows that Corollary 2 in Mania and Tevzadze (2000) does not hold in general. Note, however, that the corresponding statement in Mania and Tevzadze (2003), Corollary 3 is correct. In Section 3 we discuss variants of Claim 1.3 that are used in Biagini et al. (2000, Theorem 2.16); Hobson (2004, Theorem 3.1); Sekine (2004, Lemma A.2). These papers impose additional conditions on the underlying filtration. Since we cannot verify whether our example satisfies these extra conditions, we do not know whether it applies in their setup. At the same time, it is not obvious from the proofs in the literature that the cited statements do hold in the setup of these papers. It rather seems that the stronger condition 2 was left out accidentally in the assertions.

The paper is organized as follows. In Section 2 we construct a counterexample to Claim 1.3 based on a modification of the celebrated all-purpose example put forward by Delbaen and Schachermayer (1998). The modification achieves certain integrability needed for our purposes. The actual application of the modified example is non-trivial because our construction starts under a risk-neutral measure $Q$ rather than the objective measure $P$. In Section 3 we relate Proposition 1.2 to alternative characterizations of the VOMM from the literature.

We use the notation of Jacod and Shiryaev (2003). In particular, $\mathscr{M}, \mathscr{M}_{\text {loc }}$ denote the sets of uniformly integrable martingales and of local martingales, respectively. If these properties refer to some probability measure $Q$ rather than $P$, we write $\mathscr{M}^{Q}, \mathscr{M}_{\text {loc }}^{Q}$. 


\section{The counterexample}

The goal in this section is to find a continuous semimartingale $S$ on some filtered probability space $(\Omega, \mathscr{F}, \mathbf{F}, P)$ and an EMM $Q^{\star} \sim P$ with square-integrable density of the form (1.1) which is not the VOMM but satisfies 2" in Claim 1.3. For ease of exposition we work with an infinite time horizon but by a simple deterministic time change $\mathbb{R}_{+} \rightarrow[0,1]$ it could be transformed to a finite time horizon as usual (cf. Section 3).

We commence by modifying the construction of Delbaen and Schachermayer (1998). The reason for doing so is that otherwise Statement 5 in Lemma 2.1 would not hold. Let $B, W$ be independent Brownian motions and

$$
\begin{aligned}
M_{t} & :=\mathscr{E}(B)_{t}=\exp \left(B_{t}-\frac{1}{2} t\right) \\
N_{t} & :=\mathscr{E}(W)_{t}=\exp \left(W_{t}-\frac{1}{2} t\right)
\end{aligned}
$$

Define stopping times

$$
\begin{aligned}
\sigma & :=\inf \left\{t \geq 0: N_{t}=2\right\} \\
\tau & :=\inf \left\{t \geq 0: M_{t}=\frac{1}{2} \wedge N_{t}\right\}
\end{aligned}
$$

It is easy to see that $\tau$ is almost surely finite. Indeed, we have

$$
\begin{aligned}
\tau & =\inf \left\{t \geq 0: B_{t}-\frac{1}{2} t \leq \log \frac{1}{2} \wedge\left(W_{t}-\frac{1}{2} t\right)\right\} \\
& =\inf \left\{t \geq 0: B_{t}-W_{t} \leq 0 \text { and } B_{t} \leq \frac{1}{2} t+\log \frac{1}{2}\right\}
\end{aligned}
$$

The second condition in the second line holds for any $t$ which is large enough, the first condition is met again and again because any Brownian motion returns to 0 infinitely often. Finally define stopped processes

$$
\begin{aligned}
X & :=M^{\sigma \wedge \tau} \\
Y & :=N^{\sigma \wedge \tau} .
\end{aligned}
$$

Lemma 2.1 $X$ and $Y$ defined above have the following properties.
1. $X \in \mathscr{M}_{\text {loc }}$
2. $X \notin \mathscr{M}$
3. $Y \in \mathscr{M}$
4. $X Y \in \mathscr{M}$ (and hence $E\left(X_{\infty} Y_{\infty}\right)<\infty$ )
5. $E\left(\frac{X_{\infty}}{Y_{\infty}}\right)<\infty$
6. $\frac{Y}{X}$ is a bounded submartingale. 
ProOF. 1. This is obvious.

2. We start by showing that

$$
E\left(M_{\sigma \wedge \tau} 1_{\{\sigma<\infty\}}\right)=P(\sigma<\infty) .
$$

The argument is basically the same as in Delbaen and Schachermayer (1998) but one has to argue a little more formally. For continuous functions $\alpha: \mathbb{R}_{+} \rightarrow \mathbb{R}_{+}$set

$$
\begin{aligned}
\tau^{\alpha} & :=\inf \left\{t \geq 0: M_{t} \leq \frac{1}{2} \wedge \alpha(t)\right\} \\
\sigma^{\alpha} & :=\inf \{t \geq 0: \alpha(t)=2\} .
\end{aligned}
$$

For fixed $\alpha$ we have that $\tau^{\alpha}$ is a stopping time and $\sigma^{\alpha} \in \mathbb{R} \cup\{\infty\}$ is deterministic. Consequently,

$$
\begin{aligned}
E\left(M_{\sigma \wedge \tau} 1_{\{\sigma<\infty\}}\right) & =E\left(M_{\sigma^{N} \wedge \tau^{N}} 1_{\left\{\sigma^{N}<\infty\right\}}\right) \\
& =\int E\left(M_{\sigma^{N} \wedge \tau^{N}} 1_{\left\{\sigma^{N}<\infty\right\}} \mid N=\alpha\right) P^{N}(d \alpha) \\
& =\int E\left(M_{\sigma^{\alpha} \wedge \tau^{\alpha}} 1_{\left\{\sigma^{\alpha}<\infty\right\}} \mid N=\alpha\right) P^{N}(d \alpha) \\
& =\int E\left(M_{\sigma^{\alpha} \wedge \tau^{\alpha}}\right) 1_{\left\{\sigma^{\alpha}<\infty\right\}} P^{N}(d \alpha) \\
& =\int 1_{\left\{\sigma^{\alpha}<\infty\right\}} P^{N}(d \alpha) \\
& =\int 1_{\left\{\sigma^{N}<\infty\right\}} d P \\
& =P(\sigma<\infty),
\end{aligned}
$$

where $P^{N}$ denotes the law of $N$ on the space of continuous functions. The fourth equation follows from the independence of $M$ and $N$. The fifth equation holds because $M$ is a martingale with $M_{0}=1$ and $\sigma^{\alpha} \wedge \tau^{\alpha}$ is a bounded stopping time for fixed $\alpha$.

Now we can proceed with the proof of Statement 2. Indeed, we have

$$
\begin{aligned}
E\left(X_{\infty}\right) & =E\left(M_{\sigma \wedge \tau}\right) \\
& =E\left(M_{\sigma \wedge \tau} 1_{\{\sigma=\infty\}}\right)+E\left(M_{\sigma \wedge \tau} 1_{\{\sigma<\infty\}}\right) \\
& \leq \frac{1}{2} P(\sigma=\infty)+P(\sigma<\infty) \\
& <P(\sigma=\infty)+P(\sigma<\infty) \\
& =1=X_{0},
\end{aligned}
$$

which implies that $X$ is not a uniformly integrable martingale.

3. $Y$ is a bounded local martingale and hence a uniformly integrable martingale.

4. By independence of $B, W$ we have that $X Y$ is a positive local martingale and hence 
a supermartingale. It remains to be shown that $E\left(X_{\infty} Y_{\infty}\right)=1$. This follows from

$$
\begin{aligned}
E\left(X_{\infty} Y_{\infty}\right) & =E\left(M_{\sigma \wedge \tau} E\left(N_{\sigma} \mid \mathscr{F}_{\sigma \wedge \tau}\right)\right) \\
& =E\left(E\left(M_{\sigma \wedge \tau} N_{\sigma} \mid \mathscr{F}_{\sigma \wedge \tau}\right)\right) \\
& =E\left(M_{\sigma \wedge \tau} N_{\sigma}\right) \\
& =E\left(2 M_{\sigma \wedge \tau} 1_{\{\sigma<\infty\}}\right) \\
& =2 E\left(1_{\{\sigma<\infty\}}\right) \\
& =E\left(N_{\sigma}\right)=1 .
\end{aligned}
$$

Here, the first and the last equality hold because $N^{\sigma}$ is a bounded and hence uniformly integrable martingale. The fifth equality follows from (2.1).

5. We have

$$
\frac{X_{\infty}}{Y_{\infty}}= \begin{cases}\frac{1}{2} M_{\sigma} & \text { on } \sigma \leq \tau \\ \frac{\frac{1}{2} \wedge N_{\tau}}{N_{\tau}} \leq 1 & \text { on } \tau \leq \sigma .\end{cases}
$$

Since $M$ is a positive martingale, we have $E\left(M_{\sigma}\right) \leq M_{0}=1<\infty$ (cf. e.g. Jacod and Shiryaev 2003, I.1.39). Consequently, Statement 5 holds.

6. Since $N / M=\exp (W-B)$ is a submartingale, the same is true for $Y / X$. The boundedness follows from

$$
\frac{Y_{t}}{X_{t}}=\frac{N_{t}}{M_{t}} \leq \frac{N_{t}}{\frac{1}{2} \wedge N_{t}} \leq 1 \vee 2 N_{t} \leq 4
$$

for $t \leq \tau \wedge \sigma$.

We apply the previous lemma in order to construct a counterexample to Claim 1.3. Note, however, that we start with a probability measure $Q$ rather than $P$, i.e. $B, W$ are supposed to be $Q$-Brownian motions, Lemma 2.1 holds under $Q$ etc. Measure $P$ will only be defined later. Set $S:=X$ and define probability measures $Q^{\star} \sim Q, P \sim Q$ in terms of their Radon-Nikodym densities

$$
\frac{d Q^{\star}}{d Q}:=Y_{\infty}, \quad \frac{d P}{d Q}:=\frac{Y_{\infty}}{c X_{\infty}} \text { with } c=E_{Q}\left(\frac{Y_{\infty}}{X_{\infty}}\right) .
$$

By Statement 6 of Lemma 2.1 we have $1 \leq c<\infty$.

We now take the perspective of $P$ as given probability measure. From Statements 1-3 in the following lemma we conclude that $Q^{\star}$ satisfies Condition 1 and 2 " in the previous section. Nevertheless, it is not the VOMM by Statement 7. It is a natural and interesting albeit open question what the true VOMM looks like in the present example.

Lemma 2.2 The process $X$ and measures $P, Q, Q^{\star}$ defined in (2.2) have the following properties. 
1.

$$
\frac{d Q^{\star}}{d P}=\frac{1+1 \cdot X_{\infty}}{E_{P}\left(1+1 \cdot X_{\infty}\right)}
$$

2. $\frac{d Q^{\star}}{d P} \in L^{2}(P)$

3. $X \in \mathscr{M}^{Q^{\star}}$ (and hence $1 \cdot X \in \mathscr{M}^{Q^{\star}}$ )

4. $\frac{d Q}{d P} \in L^{2}(P)$

5. $X \in \mathscr{M}_{\mathrm{loc}}^{Q}$

6. $X \notin \mathscr{M}^{Q}$ (and hence $1 \cdot X \notin \mathscr{M}^{Q}$ )

7. $Q^{\star}$ is not the VOMM (relative to $P$ ).

8. If we assume the filtration to be generated by $(B, W)$, then all $P$-local martingales are continuous.

Proof. 1.

$$
\frac{d Q^{\star}}{d P}=\frac{d Q^{\star}}{d Q} / \frac{d P}{d Q}=c X_{\infty}=c\left(1+1 \cdot X_{\infty}\right)
$$

2.

$$
E_{P}\left(\left(\frac{d Q^{\star}}{d P}\right)^{2}\right)=E_{Q^{\star}}\left(\frac{d Q^{\star}}{d P}\right)=E_{Q}\left(\frac{d Q^{\star}}{d P} \frac{d Q^{\star}}{d Q}\right)=c E_{Q}\left(X_{\infty} Y_{\infty}\right)<\infty
$$

3. This holds because $X Y$ is a uniformly integrable $Q$-martingale (cf. Lemma 2.1(4)). 4.

$$
E_{P}\left(\left(\frac{d Q}{d P}\right)^{2}\right)=E_{Q}\left(\frac{d Q}{d P}\right)=c E_{Q}\left(\frac{X_{\infty}}{Y_{\infty}}\right)<\infty
$$

5. see Lemma 2.1(1)

6. see Lemma 2.1(2)

7. For $\varepsilon \in[0,1]$ set $\bar{Q}=(1-\varepsilon) Q^{\star}+\varepsilon Q$. Then $\bar{Q}$ is a martingale measure whose density is in $L^{2}(P)$. We have

$$
\begin{aligned}
E_{P}\left(\left(\frac{d \bar{Q}}{d P}\right)^{2}\right) & =E_{P}\left(\left(\frac{d Q^{\star}}{d P}\right)^{2}\right)+2 E_{P}\left(\frac{d Q^{\star}}{d P}\left(\frac{d \bar{Q}}{d P}-\frac{d Q^{\star}}{d P}\right)\right)+E_{P}\left(\left(\frac{d \bar{Q}}{d P}-\frac{d Q^{\star}}{d P}\right)^{2}\right) \\
& =E_{P}\left(\left(\frac{d Q^{\star}}{d P}\right)^{2}\right)+2 \varepsilon E_{P}\left(\frac{d Q^{\star}}{d P}\left(\frac{d Q}{d P}-\frac{d Q^{\star}}{d P}\right)\right)+\varepsilon^{2} E_{P}\left(\left(\frac{d Q}{d P}-\frac{d Q^{\star}}{d P}\right)^{2}\right) \\
& =E_{P}\left(\left(\frac{d Q^{\star}}{d P}\right)^{2}\right)+2 \varepsilon c\left(E_{Q}\left(X_{\infty}\right)-E_{Q^{\star}}\left(X_{\infty}\right)\right)+\varepsilon^{2} E_{P}\left(\left(\frac{d Q}{d P}-\frac{d Q^{\star}}{d P}\right)^{2}\right) .
\end{aligned}
$$

The last term is finite because $\frac{d Q}{d P}, \frac{d Q^{\star}}{d P}$ have finite $L^{2}(P)$-norm. Since $X$ is in $\mathscr{M}^{Q^{\star}}$ but not in $\mathscr{M}^{Q}$, we have $E_{Q^{\star}}\left(X_{\infty}\right)=1$ and $E_{Q}\left(X_{\infty}\right)<1$. Consequently, we have

$$
E_{P}\left(\left(\frac{d \bar{Q}}{d P}\right)^{2}\right)<E_{P}\left(\left(\frac{d Q^{\star}}{d P}\right)^{2}\right)
$$

for $\varepsilon$ small enough.

8. The filtration is generated by a bivariate $Q$-Brownian motion. According to the martingale representation thereom every $Q$-local martingale is continuous because it can be written as stochastic integral relative to $(B, W)$. In particular, the density process $Z$ of $P$ relative to $Q$ is continuous. Now let $U$ be any $P$-local martingale. This is equivalent to saying that $U Z$ is a $Q$-local martingale (cf. e.g. Jacod and Shiryaev 2003, III.3.8). In particular, $U Z$ and hence also $U$ is continuous. 


\section{Alternative characterizations of the VOMM}

In this section we review other, more explicit characterizations of the VOMM, which involve an admissibility condition in the spirit of Proposition 1.2. We will see that it cannot be relaxed in these cases either. For ease of exposition, we consider as before a univariate continuous semimartingale $S$ and assume $\mathscr{F}_{0}$ to be trivial. Moreover, we suppose that $T$ is finite and Assumption 1.1 is satisfied.

We denote the canonical decomposition of $S$ into a local martingale and a process of finite variation as

$$
S=S_{0}+M^{S}+B^{S} .
$$

By Schweizer (1995, Theorem 1) there exists an $S$-integrable process $\lambda$ such that

$$
B^{S}=\lambda \cdot\left[M^{S}, M^{S}\right]
$$

One can always write the processes $B^{S},\left[M^{S}, M^{S}\right]$ in integral form

$$
B^{S}=b^{S} \cdot A, \quad\left[M^{S}, M^{S}\right]=c^{S} \cdot A
$$

with some increasing semimartingale $A$ and predictable processes $b^{S}, c^{S}$. For Itô processes one typically chooses $A_{t}=t$. If $K$ denotes another special semimartingale with canonical decomposition

$$
K=K_{0}+M^{K}+B^{K}
$$

we write similarly

$$
B^{K}=b^{K} \cdot A, \quad\left[M^{K}, M^{S}\right]=c^{K S} \cdot A .
$$

To begin with we translate the characterization of Černý and Kallsen (2005) to the present setup.

Proposition 3.1 Suppose that L is a semimartingale such that

la) $L, L_{-}$are $(0,1]$-valued,

1b) $L_{T}=1$

1c) semimartingale $K$ defined by $L=L_{0} \mathscr{E}(K)$ satisfies $b^{K}=\left(b^{S}+c^{K S}\right)^{2} / c^{S}$,

2a) $a:=\left(b^{S}+c^{K S}\right) / c^{S}$ is S-integrable,

$2 b)$ the local martingale $Z^{Q^{\star}}:=\mathscr{E}(K) \mathscr{E}(-a \cdot S)$ is a martingale,

$2 c)$ the $Q$-local martingale $\mathscr{E}(-a \cdot S)$ is a $Q$-martingale for any EMM $Q$ with squareintegrable density.

Then $Q^{\star}$ with density $\frac{d Q^{\star}}{d P}:=Z_{T}^{Q^{\star}}$ is the VOMM. 
Proof. Conditions 1c), 2a) and integration by parts yield that $Z^{Q^{\star}}$ and $Z^{Q^{\star}} S$ are local martingales. Condition $2 \mathrm{~b}$ ) implies that $Q^{\star}$ is an EMM with density process $Z^{Q^{\star}}$. In particular, we have

$$
E\left(\left(\frac{d Q^{\star}}{d P}\right)^{2}\right)=E_{Q^{\star}}\left(\mathscr{E}(-a \cdot S)_{T}\right) / L_{0}<\infty
$$

because $\mathscr{E}(-a \cdot S)$ is a positive $Q^{\star}$-local martingale and hence a $Q^{\star}$-supermartingale. Define the predictable process $\varphi$ by $1-\varphi \cdot S=\mathscr{E}(-a \cdot S)$. The assertion follows now from Proposition 1.2.

Černý and Kallsen (2005) show that the process $L$ above always exists (provided that Assumption 1.1 holds). $a$ is called adjustment process and $L$ is the so-called opportunity process, i.e. $L_{t}^{-1}-1$ measures the square of the maximal Sharpe ratio attainable by dynamic trading in the asset between dates $t$ and $T$ (cf. also Černý and Kallsen (2006), Theorem 2.8 in this respect). For the purposes of the above proof, one can in fact do without the condition $L \leq 1$ in 1a).

Remark 3.2 The proof of Proposition 3.1 shows that the stronger condition,

$\left.2^{\prime} b\right)$ the local martingale $Z^{Q^{\star}}$ is a square-integrable martingale,

is in fact implied by conditions $1 a)-2 b$ ).

Biagini et al. (2000) and Hobson (2004) characterize the VOMM in terms of some fundamental equation. In their language Proposition 1.2 reads as follows.

Proposition 3.3 Suppose that $\eta$ is some predictable process, $R$ some local martingale with $\left[R, M^{S}\right]=0$, and c some positive number such that

1)

$$
\exp \left(\lambda \cdot B_{T}^{S}\right) \mathscr{E}(R)_{T}=c \mathscr{E}\left(\eta \cdot\left(M^{S}+2 B^{S}\right)\right)_{T}
$$

or, if $R$ is continuous (note that $R$ may generally have jumps although $S$ is supposed to be continuous in this paper),

$$
\lambda \cdot B_{T}^{S}=\eta \cdot\left(M^{S}+2 B^{S}\right)-\frac{1}{2} \eta^{2} \cdot\left[M^{S}, M^{S}\right]-R_{T}+\frac{1}{2}[R, R]_{T}+\log c,
$$

2a) $\eta$ is S-integrable,

$2 b)$ the local martingale $Z^{Q^{\star}}:=\mathscr{E}\left(-\lambda \cdot M^{S}+R\right)$ is a martingale,

2c) for $a:=\lambda-\eta$ the $Q$-local martingale $\mathscr{E}(-a \cdot S)$ is a $Q$-martingale for any EMM $Q$ with square-integrable density.

Then $Q^{\star}$ with density $\frac{d Q^{\star}}{d P}:=Z_{T}^{Q^{\star}}$ is the VOMM. 
PROOF. The existence of the stochastic integrals follows from 2a). It is easy to see that (3.2) and (3.3) are equivalent for continuous $R$. Condition 2b) implies that $Q^{\star}$ is a probability measure with density process $Z^{Q^{\star}}$. It is even an EMM because $\left[R, M^{S}\right]=0$. The fundamental equation (3.2) and straightforward calculations yield

$$
Z_{T}^{Q^{\star}}=c \mathscr{E}((\eta-\lambda) \cdot S)_{T}
$$

In particular, we have that

$$
E\left(\left(\frac{d Q^{\star}}{d P}\right)^{2}\right)=c E_{Q^{\star}}\left(\mathscr{E}((\eta-\lambda) \cdot S)_{T}\right)<\infty
$$

because $\mathscr{E}((\eta-\lambda) \cdot S)$ is a positive $Q^{\star}$-local martingale and hence a $Q^{\star}$-supermartingale. Defining $\varphi$ by $1+\varphi \cdot S=\mathscr{E}((\eta-\lambda) \cdot S)$, the assertion follows from Proposition 1.2.

Remark 3.4 Given processes $a, K, L$ that satisfy conditions of Proposition 3.1 one contructs the processes of Proposition 3.3 as follows

$$
\begin{aligned}
\eta & =-\frac{c^{K S}}{c^{S}} \\
R & =M^{K}+\eta \cdot M^{S} \\
c & =1 / L_{0} .
\end{aligned}
$$

Conversely, given $\eta, R, c$ that satisfy conditions of Proposition 3.3 the processes $a, K, L$ of Proposition 3.1 can be obtained from

$$
\begin{aligned}
a & =\lambda-\eta, \\
K & =-\lambda \cdot M^{S}+R+a \cdot S+\left(a^{2} c^{S}-a b^{S}\right) \cdot A, \\
L & =\mathscr{E}(K) / c .
\end{aligned}
$$

Biagini et al. (2000, Theorem 2.16) leave out Condition 2c) and instead impose the condition that $Z_{T}^{Q^{\star}}$ is square integrable, which follows already from Conditions 1), 2a) and 2b) (cf. Remark 3.2). They require, in addition, that the filtration is a product of a $P$ Brownian motion filtration with another filtration generated by a finite-dimensional (but not necessarily continuous) $P$-martingale. Hobson (2004, Theorem 3.1) replaces 2c) with the requirement that $\mathscr{E}(-a \bullet S)$ is a $Q^{\star}$-martingale, i.e. with Condition 2"c) below. He assumes in addition that the filtration is generated by two independent $P$-Brownian motions.

Proposition 1.2 in the language of Sekine (2004, Lemma A.2) reads:

Proposition 3.5 Suppose that a is a predictable process and $R$ a local martingale with $\left[R, M^{S}\right]=0$ such that

1) the local martingale $Z^{Q^{\star}}:=\mathscr{E}\left(-\lambda \cdot M^{S}+R\right)$, satisfies

$$
\frac{Z_{T}^{Q^{\star}}}{Z_{t}^{Q^{\star}}}=V_{t} \frac{\mathscr{E}(-a \cdot S)_{T}}{\mathscr{E}(-a \cdot S)_{t}}, \quad t \in[0, T],
$$


where

$$
V_{t}:=E\left(\left(\frac{Z_{T}^{Q^{\star}}}{Z_{t}^{Q^{\star}}}\right)^{2} \mid \mathscr{F}_{t}\right)
$$

2a) a is S-integrable,

$\left.2^{\prime} b\right) Z^{Q^{\star}}$ is a square-integrable martingale,

2c) the $Q$-local martingale $\mathscr{E}(-a \cdot S)$ is a $Q$-martingale for any EMM $Q$ with squareintegrable density.

Then $Q^{\star}$ with density $\frac{d Q^{\star}}{d P}:=Z_{T}^{Q^{\star}}$ is the VOMM.

ProOF. $Q^{\star}$ is a probability measure with density process $Z^{Q^{\star}}$. It is even an EMM because $\left[R, M^{S}\right]=0$. Define the predictable process $\varphi$ by $1-\varphi \cdot S=\mathscr{E}(-a \cdot S)$. From (3.4) for $t=0$ we obtain (1.1). The assertion follows now from Proposition 1.2.

Remark 3.6 The opportunity process L of Proposition 3.1 corresponds to $1 / V$ in Proposition 3.5.

Sekine (2004, Lemma A.2) leaves out Condition 2c) but, in line with Hobson (2004), requires additionally that the filtration is generated by two independent $P$-Brownian motions. We note that the Conditions 1), 2a) and 2'b) already imply Condition 2"c) below. Indeed, from (3.4) it follows that $Z^{Q^{\star}}$ equals $\mathscr{E}(-a \cdot S) / V$ up to some constant. By (3.5), $V\left(Z^{Q^{\star}}\right)^{2}$ is a martingale, which in turn implies $2 " \mathrm{c})$.

Let us turn back to the counterexample from Section 2 to demonstrate that it applies also in the more structured environment of Propositions 3.1, 3.3 and 3.5. To this end we formulate a weakened version of the admissibility condition $2 \mathrm{c}$ ),

2"c) the $Q^{\star}$-local martingale $\mathscr{E}(-a \cdot S)$ is a $Q^{\star}$-martingale.

Proposition 3.7 Propositions 3.1, 3.3 and 3.5 do not hold if the Condition 2c) is replaced with the weaker Condition 2"c).

PROOF. We formulate the proof in the language of Proposition 3.1. The proof for Propositions 3.3, 3.5 is straightforward, using the definitions of $\eta, R, V$ and $c$ below.

Consider the processes $X, Y$ of Lemma 2.1 constructed under measure $Q$, and define the measures $P, Q^{\star}$ and the constant $c$ as in (2.2). Denote by $Z$ the uniformly integrable $Q$-martingale with terminal value $Y_{\infty} / X_{\infty}$. Since the results in this section require $T$ to be finite, we consider the time-changed filtration $\widetilde{\mathscr{F}}_{t}:=\mathscr{F}_{\tan \frac{\pi t}{2 T}}$ and time-changed processes $\widetilde{X}_{t}:=X_{\tan \frac{\pi t}{2 T}}$ etc. for $t \in[0, T]$. By abuse of notation we denote the time-changed processes $\widetilde{X}, \widetilde{Y}, \widetilde{Z}$ again by $X, Y, Z$. One easily verifies that the statements of Lemmas 2.1 and 2.2 remain true for these processes with $\infty$ replaced by $T$. 
Define

$$
\begin{gathered}
S:=X, \quad a:=-\frac{1}{X}, \quad L:=\frac{Y}{X Z}, \quad K:=\frac{1}{L} \cdot L, \\
\eta:=\lambda-a=-\frac{c^{K S}}{c^{S}}, \quad R:=M^{K}+\eta \cdot M^{X}, \quad V:=\frac{1}{L} .
\end{gathered}
$$

We have $L>0, L_{T}=1$ and by Statement 6 of Lemma $2.1 L \leq 1$, hence $L$ meets conditions 1a), 1b). The process $\mathscr{E}(K) \mathscr{E}(-a \cdot S)=Z_{0} Y / Z$ is a $P$-martingale if and only if $Y$ is a $Q$-martingale. The latter holds by construction (cf. Statement 3 of Lemma 2.1), hence condition $2 \mathrm{~b}$ ) is satisfied. Yor's formula and zero drift condition under $P$ yield

$$
0=B^{K}-a \cdot B^{S}-a \cdot[K, S]
$$

By construction (cf. Statement 3 of Lemma 2.2) $\mathscr{E}(-a \cdot S)=X \in \mathscr{M}^{Q^{\star}}$ and the Girsanov theorem yields

$$
B^{S}+[K, S]-a \cdot[S, S]=0 .
$$

Equations (3.6) and (3.7) imply

$$
\begin{aligned}
B^{K} & =a^{2} \cdot[S, S], \\
a & =\frac{b^{S}+c^{K S}}{c^{S}} .
\end{aligned}
$$

Hence conditions 1c), 2a) and 2"c) are satisfied as well. However, by Statement 7 of Lemma $2.2 Q^{\star}$ is not the VOMM.

\section{References}

Biagini, F., P. Guasoni, and M. Pratelli (2000). Mean-variance hedging for stochastic volatility models. Mathematical Finance 10(2), 109-123.

Černý, A. and J. Kallsen (2005). On the structure of general mean-variance hedging strategies. SSRN working paper, http://ssrn.com/abstract=882762.

Černý, A. and J. Kallsen (2006). Mean-variance hedging and optimal investment in Heston's model with correlation. SSRN working paper, http://ssrn.com/abstract=909305.

Delbaen, F., P. Monat, W. Schachermayer, M. Schweizer, and C. Stricker (1997). Weighted norm inequalities and hedging in incomplete markets. Finance \& Stochastics 1, 181-227.

Delbaen, F. and W. Schachermayer (1996). The variance-optimal martingale measure for continuous processes. Bernoulli 2, 81-105.

Delbaen, F. and W. Schachermayer (1998). A simple counterexample to several problems in the theory of asset pricing. Mathematical Finance 8, 1-11.

Hobson, D. (2004). Stochastic volatility models, correlation, and the $q$-optimal martingale measure. Mathematical Finance 14, 537-556. 
Jacod, J. and A. Shiryaev (2003). Limit Theorems for Stochastic Processes (second ed.). Berlin: Springer.

Mania, M. and R. Tevzadze (2000). A semimartingale Bellman equation and the varianceoptimal martingale measure. Georgian Mathematical Journal 7, 765-792.

Mania, M. and R. Tevzadze (2003). A semimartingale backward equation and the variance-optimal martingale measure under general information flow. SIAM Journal on Control and Optimization 42, 1703-1726.

Pham, H. (2000). On quadratic hedging in continuous time. Mathematical Methods of Operations Research 51, 315-339.

Schweizer, M. (1994). Approximating random variables by stochastic integrals. The Annals of Probability 22, 1536-1575.

Schweizer, M. (1995). On the minimal martingale measure and the Föllmer-Schweizer decomposition. Stochastic Analysis and Applications 13, 573-599.

Schweizer, M. (2001). A guided tour through quadratic hedging approaches. In E. Jouini, J. Cvitanic, and M. Musiela (Eds.), Option Pricing, Interest Rates and Risk Management, pp. 538-574. Cambridge: Cambridge University Press.

Sekine, J. (2004). On the computation of $L^{2}$-hedging strategy with stochastic volatility. Unpublished manuscript. 